\title{
Entrelacs
}

Cinéma et audiovisuel

Jodorowsky, d'un art à l'autre

\section{Voies multiples, voies d'accès à la totalité}

\section{Émilie Combes}

\section{OpenEdition}

Journals

Édition électronique

URL : http://journals.openedition.org/entrelacs/5639

DOI : 10.4000/entrelacs.5639

ISSN : 2261-5482

Éditeur

Éditions Téraèdre

\section{Référence électronique}

Émilie Combes, « Voies multiples, voies d'accès à la totalité », Entrelacs [En ligne], 16 | 2019, mis en ligne le 06 novembre 2019, consulté le 05 décembre 2019. URL : http://journals.openedition.org/ entrelacs/5639; DOI : 10.4000/entrelacs.5639

Ce document a été généré automatiquement le 5 décembre 2019

Tous droits réservés 


\title{
Voies multiples, voies d'accès à la totalité
}

\author{
Émilie Combes
}

\section{Introduction}

1 Cinéaste $^{1}$, mime, scénariste de bandes dessinées, romancier, poète, ou encore théoricien de tarot divinatoire, Alejandro Jodorowsky est l'auteur d'une œuvre abondante et protéiforme et se dit «multiple ${ }^{2}$ ». Son goût pour le spectacle vivant se concrétise lors de la création avec Fernando Arrabal et Roland Topor du groupe Panique en 1962, qui lui permet de réaliser des "éphémères paniques ", performances artistiques souvent reçues comme provocantes et scandaleuses, desquelles se dégage une atmosphère festive, cérémoniale, à la fois mystique et onirique. Cette esthétique du multiple, à travers laquelle il exhibe dans ses œuvres l'homme tel qu'il est, odieux ou monstrueux, mais aussi capable de beauté et de poésie, semble s'être poursuivie, adoucie par la sagesse du temps, dans ses deux derniers films La Danza de la realidad (2013) et Poesía sin fin (2016), où il ne cesse d'explorer diverses voies d'expression et nous donne accès à un univers intime et poétique. Dans la mesure où son œuvre interroge constamment les relations entre les arts, nous étudierons les dialogues qui se tissent au cœur de ces films entre diverses formes artistiques et influences culturelles ou biographiques, pour explorer en quoi le cinéma de Jodorowsky se construit, à l'instar de toutes les créations paniques, comme un art total, lieu de la pluralité où se trouvent mêlées diverses pratiques artistiques et où le langage corporel, à travers les arts populaires ou encore la question de la démesure, tient une place prépondérante. Il s'agira enfin d'observer la manière dont Jodorowsky propose une sorte d'autobiographie fantasmée et thérapeutique. 


\section{Le cinéma comme lieu de la pluralité : une « fête- spectacle »}

2 Le cinéma est rapidement devenu pour Jodorowsky un lieu privilégié des manifestations paniques qui, tendant à la "fête-spectacle ${ }^{3}$ ", ne rejettent nullement les images fortes ou violentes, voyant comme une nécessité le fait d'agir sur le spectateur par les retombées scéniques de leurs œuvres. En effet, dès la création de Panique au début des années 1960, les créations du groupe privilégient une attitude plastique concrète : elles ne doivent pas "exprimer » un acte, mais le commettre, au point que pour Jodorowsky, la langue écrite ne peut être panique à moins de "s'intégrer dans un ensemble corporel, vocal, spectaculaire ${ }^{4}$. " En effet, prenant conscience de la limite des happenings, les artistes paniques orientent leurs créations vers des manifestations susceptibles de s'intégrer aisément dans les normes en vue de les faire éclater. Remettant en cause le théâtre sclérosé de l'époque et la stérilité artistique dans laquelle il est enfermé, Jodorowsky propose de nouvelles possibilités sur lesquelles fonder l'acte théâtral. Cette nouvelle conception du spectacle vivant va de pair pour Panique avec une redécouverte du corps, pensé comme moyen de communication et comme matérialité propre à constituer l'événement scénique sur les planches. Jodorowsky propose une création qui prend ses distances avec le texte et prône au contraire l'acte. Il conçoit la création artistique de manière plus concrète, dans laquelle seraient employés des morceaux de la réalité, subordonnés à la création plastique. Il ne s'agit donc plus de figurer mais de laisser la possibilité au public de palper le réel. Dès lors, ses créations intègrent toutes sortes de matériaux, notamment les éléments organiques comme lors de leur performance en mai 1965 pendant du festival de la Libre Expression ${ }^{5}$. Le dramaturge entend bannir les décors et autres «bâtardes expositions de peintures et de sculptures ${ }^{6}$ ", pour leur substituer des " objets actifs » et bien sûr, éphémères. Pour le cinéaste chilien, le cinéma est alors devenu un des lieux privilégiés de ces manifestations. Pour autant, le cinéma de Jodorowsky, tout comme ses éphémères, tente de rendre compte davantage de la vie en termes de processus, " en train d'être ", mettant en avant le corps et l'acte comme manifestation concrète d'un certain nombre de possibles, et n'en reste pas moins une création artistique, et non la vie même.

3 Les artistes paniques pensent que la tâche des fêtes-spectacle est d'éveiller le spectateur dans l'euphorie panique, face à un acteur qui cesse de jouer un personnage mais s'approche peu à peu de la personne. Cette euphorie conduit le spectateur à un état de grâce et lui permet donc de s'éveiller de sa léthargie, de prendre conscience de la pluralité de l'homme, de la poésie et de l'abjection de la vie, tant le mode d'expression choisi est concret, et donc saisissant. De la même manière que « l'éphémère panique a pour tâche d'abandonner la figuration et l'abstraction pour arriver à une manifestation concrète $^{7}$ ", le cinéma de Jodorowsky est fait à partir de moyens concrets et intègre toutes sortes de matériaux et d'éléments organiques. Ainsi, dans Poesía sin fin, les décors de la rue principale sont des photos grandeur nature en noir et blanc, mises en place directement par des «appariteurs » en noir qui ne sont pas sans rappeler les artifices du théâtre, à l'instar de l'ouvrier des pièces de Novarina qui déplace les objets. On retrouve ces hommes à divers endroits du film comme lors de l'entretien entre Jaime (interprété par Brontis Jodorowsky) et Alejandro (interprété par Adan Jodorowsky) à la maison ou lors de la partie de cartes chez la grand-mère maternelle. Cette esthétique 
qui joue sur les artifices, que l'on retrouve dans le théatre panique et l'art pauvre ${ }^{8}$, est également liée à la volonté de mettre en scène les ficelles du spectacle, afin de montrer que l'on est au cinéma.

4 Par ailleurs, dans la réflexion des paniques, les éphémères ont pour but « de libérer l'homme de ses moules quotidiens afin qu'il puisse, par l'improvisation, développer la totalité de son être ${ }^{9}$.» L'éphémère peut donc être rapproché d'une cérémonie qui a quelque chose de sacré, de primitif, exprimant avec violence les pulsions les plus souterraines qu'enferme notre âme. Il est certes une recherche artistique mais surtout humaine et spirituelle: "L'éphémère est donc à la frontière exacte entre la représentation et la présentation, entre l'irréel et le réel, entre l'imaginaire et la vie ${ }^{10}$. » Il est une «recherche à travers le geste poétique d'une certaine spiritualité qui élève l'âme ${ }^{11 .}$ » Cette recherche du geste poétique, de l'acte poétique même comme acte de rébellion contre les normes et les contraintes, se retrouve dans Poesía sin fin, lorsque le jeune Jodorowsky (Jeremias Herskovits) s'affirme comme poète en coupant l'arbre de ses ancêtres, premier "geste poétique », ou encore lorsqu'avec Enrique Linh (Leandro Taub), il décide d'aller à l'encontre des contraintes, en marchant droit à travers la ville, ou en peignant la statue de Neruda en noir pour la rendre invisible. Pour Jodorowsky, la poésie est un acte qui permet de développer sa conscience, et si l'éphémère panique décontextualise ce qui est contextuel pour nous mener vers l'euphorie panique qui est l'expression de la liberté absolue, cette libération passe par l'idée de performance dans le cadre cinématographique.

5 Selon Jean-Paul Coillard, l'acteur chez Jodorowsky ne doit plus se donner en spectacle pour qu'on le regarde, mais " établir un contact avec le mystère intérieur ${ }^{12}$ », le sien et celui du spectateur: "Le spectacle ainsi conçu cesserait dès lors de n'être qu'une simple distraction pour devenir un objet, un vecteur de connaissances, un spectacle intelligent ${ }^{13}$. » Ainsi, le cinéma de Jodorowsky se rapproche des éphémères dont le but était de faire tomber les masques du carnaval social pour parvenir à la personne réelle, être sans limite du point de vue de l'expression, et ainsi conduire à une libération :

Je crois que l'art doit libérer les forces de l'inconscient et doit travailler comme une clé dans votre inconscient. L'artiste sort tout ce qu'il a en lui-même et le met dans une œuvre d'art. Les symboles qu'il utilise doivent jouer un rôle cathartique, unir les forces dispersées dans l'inconscient et les projeter à l'extérieur de l'individu. [...] Le but de l'œuvre d'art est de canaliser toutes ces forces, de les orienter vers un point de fusion. L'artiste est un catalyseur d'énergies ${ }^{14}$.

\section{L'influence des arts populaires : exhausser le corps}

Les œuvres paniques n'hésitent donc pas à mettre en scène l'organique, le réel, et travaillent en ce sens sur la présence du corps, dans des mises en scène très ritualisées. Le geste y est souvent utilisé comme langage et le corps de l'acteur est mis en scène sans inhibition. Cet "exhaussement» du corps passe alors entre autres chez Jodorowsky par l'esthétique de la fête, des rituels et du déguisement, notamment à travers la figure des artistes-perfomeurs ou encore du monde du cirque. Le corps devient un moyen d'expression, un outil de liberté, ou encore une illustration de ce que nous sommes, entre magnificence et abjection. Dans leur volonté de joindre le réel et l'imaginaire, puisque les paniques considèrent que les deux univers s'interpénètrent et même finissent par se rencontrer complètement, la figure du clown devient privilégiée: «Les clowns, tout comme les logiques non-aristotéliciennes, comme les 
carrés de papier, ont la possibilité de changer, sont capables de se déformer, de répondre en structure, avoir une pensée multiple ${ }^{15}$.» Dans Poesía sin fin, le clown est aussi lié à l'histoire de Jodorowsky : les chaussures de clown que le personnage fait faire seraient le symbole de son souhait de revenir au monde populaire auquel il appartient, une volonté peut-être inconsciente de se rapprocher de son père.

7 Les premiers instants de La Danza de la realidad plongent le spectateur dans le monde du cirque, au milieu des clowns, jongleurs ou autres cracheurs de feu, misérables - ils vivent dans la précarité, dans la poussière du désert chilien - mais riches en couleurs et en identités, contrairement aux spectateurs présents sous le chapiteau qui portent des masques identiques faisant d'eux des silhouettes dans une masse anonyme, qui contrastent avec la cohorte de lépreux au milieu d'une aride et poussiéreuse montagne. De même, Poesía sin fin laisse la part belle au monde des circassiens dont le maître mot, comme dans La Danza de la realidad, est de se donner à l'illusion. On retrouve également dans le dernier film de Jodorowsky l'esthétique de la fête, souvent associée au déguisement ${ }^{16}$, qui rappelle celle du carnaval ou encore de L'Enterrement de la sardine ${ }^{17}$. Fidèle au principe panique de création, Jodorowsky joue sur le principe de confusion et procède à une accumulation de plans contrastés juxtaposant vision monstrueuse, mise en scène des marginaux et dimension cérémoniale. Pour autant, la réalisation reste relativement sobre et dépouillée. Le souhait du cinéaste est que « la beauté jaillisse du contenu, pas de la forme ${ }^{18}$ ", c'est pourquoi les images, tournées en numérique à la Red Epic, n'ont été que peu retravaillées - seulement quelques couleurs - pour laisser pleinement la place au contenu : «J'ai tué l'esthétisme pour créer une autre esthétique. Je me suis limité à l'essentiel ${ }^{19}$. "

8 Le corps, à la fois terrifiant et comique, exprime un paradoxe, de la même manière que la mise en scène du bas corporel, - qu'il s'agisse d'un langage gestuel décomplexé, de comportements triviaux ou de l'évocation des excreta et des besoins du corps - exprime une vision grotesque et dévoile, parfois de manière provocante, ce qu'est l'Homme, tant le corps de l'acteur reflète ce que nous sommes. Selon Peter Brook, le corps devient dans le théâtre des années 1960 un « instrument " par lequel passe tout événement, le « lieu de toutes les possibilités ${ }^{20}$ » et qui doit être traité de manière quasi cérémoniale, pour exprimer la vie. Cette mise en valeur du corps passe par un goût pour les arts populaires. Tandis que le langage apparaît comme une interface qui sépare l'homme du réel, le spectacle panique, par ce retour au corps, tente de réduire cette scission, et l'impact émotionnel sur le spectateur n'en sera que plus conséquent. Poesía sin fin explore cette idée de la "fête spectacle", en proposant même des sortes de happenings : la maison des artistes devient le lieu de performances artistiques variées entre les "ultra-pianistes" ou les "danseurs symbiotiques ${ }^{21}$ ", et les fêtes que Jodorowsky donne chez lui font intervenir les arts populaires : danseurs, jongleurs, s'y retrouvent, tandis que lui-même se livre aux arts de la marionnette, jusqu'à incarner un Pierrot ailé lors du Carnaval. Le langage passe alors beaucoup par le corps et le geste, qui se substituent à l'hégémonie de la parole pour créer une "poésie pour les sens ", une "poésie dans l'espace ${ }^{22}$ ", selon les souhaits d'Artaud. Le spectacle et les images cinématographiques qui en découlent sont donc parfois bien hétérogènes, par la diversité des moyens d'expression, qu'il s'agisse du chant, de la performance ou de l'exhibition du corps, et confèrent ainsi à son œuvre une grande richesse sensorielle.

9 Si la dimension clownesque de son œuvre ne provient pas comme chez Arrabal du comportement infantile de ses personnages, on retrouve bien l'aspect insolite de 
certains d'entre eux qui offrent parfois des scènes en décalage avec la situation, notamment dans Poesía sin fin lors du spectacle du jongleur au cours de l'enterrement qui se déroule au Café Iris. Chez Arrabal, l'univers reste davantage un univers inquiétant où grotesque et tragique s'entremêlent, malgré les jeux clownesques et l'intervention de certains intermèdes qui viennent décongestionner la tension dramatique. Dans les derniers films de Jodorowsky, la dimension insolite et grotesque de certains personnages ne sert pas à matérialiser l'horreur de situations mais plutôt à englober l'humanité dans sa totalité et à exhausser le corps dans ce qu'il a de pluriel et de poétique.

\section{Englober l'humanité dans sa totalité}

10 Ce qui distingue l'homme panique, c'est cette volonté et son aptitude à profiter de la vie, et son refus de se faire enfermer dans une pensée normative, son refus d'imaginer la réalité sans monstruosités ni transgressions. La monstruosité, tant désavouée et rejetée, va devenir un des fers de lance de la création panique, commune à Arrabal et Jodorowsky, soucieux de valoriser l'autre dans sa totalité et de reconnaître l'altérité. Ainsi, le monstrueux, que l'on repousse par crainte de la différence, va être mis en scène. Il n'est plus question d'avertir - monstrum étant un dérivé de monere qui signifie " avertir ", « faire savoir »-, de faire du monstre un signe nous rappelant les dangers et les menaces, mais bien au contraire de montrer - monstrare - l'autre. Et tout l'intérêt et la richesse du "monstre" réside dans une hésitation essentielle, qui veut que le monstre soit à la fois transgression à l'ordre du monde, contre-nature, et en un sens, unique, exception. L'œuvre des paniques, fascinés par la puissance évocatrice et artistique des monstres, est parsemée de figures monstrueuses, d'êtres anormaux. Jodorowsky affirmait à ce sujet :

J'ai toujours aimé les monstres : pour moi, ils représentent l'imagination biologique de la nature, et j'aime tout ce qui n'est pas la normalité. Ça m'attire et je vais voir ce que c'est, comme quelque chose d'artistique ${ }^{23}$.

11 Dans ses deux derniers films se succèdent de nombreux avatars de cette " anormalité ", artistes ambulants appartenant à l'univers du cirque, nains, personnages mutilés, infirmes, ou individus en marge de la communauté et de la ville, comme autant d'échos provenant d'une enfance faite d'images traumatisantes - «Les infirmes étaient déjà là quand j'étais enfant. Le village était rempli d'hommes mutilés par les accidents de la mine et les explosions à la dynamite ${ }^{24}$ »-, ou d'un besoin de laisser place à d'autres possibles, à la marge. Une idée de marginalité qui se retrouve dans le symbole même de Tocopilla, la ville natale de Jodorowsky. Rivée entre une montagne aride et la mer, elle présente une allure fantomatique et misérable. Dès les premières images de La Danza de la realidad, on retrouve le motif du désert, cher à Jodorowsky dans ses premières œuvres cinématographiques. Si l'immense étendue de roches, vide et stérile, ne vient plus symboliser ici le reflet de l'intérieur du héros, de son âme, le spectateur ne peut s'empêcher de considérer cette zone désertique dans laquelle évoluent les pestiférés comme le symbole d'une exclusion de la vie sociale, un lieu d'ascèse, de marginaux. De même, le personnage mi-réel mi-onirique de Théosophe (Cristobal Jodorowsky) à qui Jaime interdit à son fils de parler, vit au milieu des pierres du port et se trouve isolé du reste de la ville. Et pour autant, dans Poesía sin fin, c'est un ivrogne qui apporte au jeune Alejandro la révélation poétique. 
12 Si Jodorowsky voue un si grand intérêt et un attachement à la représentation des marginaux, c'est que, tout comme Arrabal, lui-même a souffert de cette situation d'exclusion dans sa jeunesse. Nombreuses sont les scènes dans La Danza de la realidad où il se trouve rejeté par les autres enfants de son âge parce qu'il a la peau blanche et qu'il est juif. Ses camarades de classe le surnomment "Pinocchio ", en référence à la forme de son nez, et rient de son pénis en forme de "champignon ». Ironiquement, le réalisateur est devenu pour les habitants une sorte de sauveur grâce au tournage du film qui a permis quelques rénovations dans la ville : «Je suis le héros qui a apporté le filtre magique pour sauver son peuple, et ce filtre magique c'est le cinéma ${ }^{25}$." Dans Poesía sin fin, le jeune Alejandro, associé à un homosexuel par son père parce qu'il se destine à la création poétique, est également vu comme un rebu, et pour écrire, il est contraint de se mettre lui-même à la marge en quittant la demeure familiale. Paradoxalement, ces états de marginalité permettent un rapport privilégié avec sa propre conscience. Le monstrueux est tout ce qui, dans une société donnée, en fonction de ce qu'elle prône et met en valeur, est considéré comme différent et fait peur par sa "non-conformité ». Il impressionne et effraye en raison de l'ignorance qu'on a de lui. Dans le monde de Jodorowsky, les monstres sont donc aussi bien les êtres difformes que l'homosexuel, l'opposant idéologique, ou bien celui qui ne sait pas s'adapter au système. Dès lors, par la marginalisation assumée, les personnages parviennent à vivre libérés des normes auxquelles ils ne correspondent pas et créent leur propre « normalité » en présentant d'autres manières de vivre.

13 Le corps devient également la scène de toutes les abjections. Il est abject en ce qu'il est dégradé. Le difforme, l'infirme sont perçus comme monstrueux en ceci qu'ils donnent immédiatement à voir une anomalie et, par là même, expriment la brisure de l'ordre. Et pourtant, le « monstre » est présenté comme un être naturel. À l'image de la difformité s'ajoute également l'idée de l'abjection physique, due aux excreta du corps humain, comme la naine qui a ses règles, mais dont le sang est vu comme sacré. Dans le principe panique d'englober l'humanité dans sa totalité, cette primauté accordée aux personnages «hors normes» montre la nécessité d'accepter l'abjection humaine, traditionnellement exclue de la vision sublimée de la littérature et de l'art. La valorisation de la différence prend dès lors une dimension éthique. Pour l'ordre social, tous ceux qui présentent une différence d'apparence physique, de sensibilité ou de comportement doivent être neutralisés ou ghettoïsés. Les paniques offrent un refuge à ces «monstres ", et en suscitant la curiosité du spectateur ils visent ainsi à provoquer un questionnement vis-à-vis des normes.

14 Au spectateur donc de faire l'effort de voir au-delà. Mettre en avant le marginal, ce que l'on rejette par inadéquation, est un moyen supplémentaire pour les artistes paniques de manifester leur intérêt pour l'homme dans sa totalité. Ce qui est perturbant, c'est en effet l'idée que le monstre n'est pas substantiellement différent de nous, qu'il est un reflet d'une partie de notre intériorité. Le «double monstrueux ${ }^{26}$ » selon René Girard est l'Autre dans tout ce qu'il a de semblable et de différent avec nous. Selon Michel Viegnes, «voir le monstre comme une projection de l'angoisse existentielle que nous portons en nous » est quelque peu réducteur et excessif, mais on pourrait penser en revanche que «la force perturbatrice du monstrueux trouve sa source [...] dans l'incertitude identitaire qui nous taraude et l'angoisse profonde que suscite en nous ce ventre mou, là où nous souhaiterions un noyau dur ${ }^{27}$. " 
La mise en scène du marginal fonctionne alors comme un vecteur d'acceptation du «monstre » qui est en nous, dans une perpétuelle quête identitaire. En mettant en scène les sujets d'une aversion comme un "double monstrueux » de soi, Jodorowsky contraint l'esprit à accepter ce qui est étranger à lui-même. En accord avec le premier sens du verbe monere, dont le substantif monstrum est dérivé, le monstre permet donc de faire songer à quelque chose, d'ouvrir les portes de connaissances nouvelles et de décentrer notre regard.

Cette mise en scène du multiple, cette volonté de laisser place à la pluralité et au tout présent dans l'appellation même de Panique -, dans une constante (con)fusion entre le sublime et le grotesque, pierre angulaire de panique, confirme bien le lien entre le cinéma de Jodorowsky et les principes paniques dont les créations avaient pour but de permettre à l'homme d'abandonner sa construction sociale pour retrouver sa nature essentielle. On retrouve dans l'œuvre du cinéaste cette même recherche artistique, à travers le geste poétique, mais surtout une quête humaine et spirituelle, faisant vivre à ses personnages une voie initiatique, qui agirait sur le spectateur comme un électrochoc. L'attitude panique rejette de façon systématique tout ce qui est restrictif, cherchant par le fait même à dissoudre les oppositions. Aussi dans Poesía sin fin, Stella Diaz (Pamela Flores) fait-elle appel à toute la complexité intellectuelle, sensorielle, animale et spirituelle qui est en chacun de nous. Figure haute en couleur avec ses formes voluptueuses, sa colossale chevelure rouge vif, ses jambes peintes aux couleurs de l'arc en ciel et son lourd manteau de fourrure, Stella représente cette association du sublime et du grotesque, du fascinant et du trivial - à la fois grande poétesse, muse de Jodorowsky, mais capable de se battre vulgairement avec des hommes dans un bar sordide, à moitié nue -, et elle constitue une rencontre nécessaire au jeune poète dans son cheminement initiatique, dans sa quête de soi. Après avoir longtemps été fasciné par son personnage, il lui est nécessaire de rompre avec elle pour se trouver lui-même, ne plus être qu'un simple reflet. Cette pluralité s'inscrit dans la logique panique de complémentarité plurielle où la confusion devient un principe structurant.

\section{L'œuvre cinématographique comme autobiographie fantasmée}

17 Jodorowsky ne cesse de transformer dans son œuvre une "mémoire familiale en légende héroïque ${ }^{28} »$, proposant une sorte d'autobiographie fantasmée et thérapeutique. On retrouve en ce sens dans son œuvre l'héritage de la pensée surréaliste - jusqu'à croiser le personnage d'André Breton dans Poesía sin fin -, attachée à l'importance de «libérer l'homme de l'emprise de la raison, des contraintes utilitaires, d'ordre moral, social, esthétique des siècles passés en mettant en œuvre le jeu désintéressé de la pensée, les forces obscures de l'inconscient ${ }^{29}$." En effet, Jodorowsky ne présente pas dans ses films une fade reconstitution de son enfance, mais livre une vision onirique de ses jeunes années ( $L a$ Danza de la realidad) et de sa « construction » de poète (Poesía sin fin) dans laquelle s'entremêlent subtilement réalité et fantasmes, vie réelle et vision idéale conçue dans l'imagination de son esprit d'enfant et de jeune homme. 


\section{Sublimer la réalité}

18 Dans la discrétion la plus totale, "sans interview, ni making-of, ni photos, ni promotion $^{30} »$, Alejandro Jodorowsky est retourné dans la ville de son enfance pour y tourner cette autobiographique fictive. C'est donc à Tocopilla, ville du nord du Chili où le réalisateur a vu le jour en février 1929, que le film débute. Fuyant leur Russie natale et les pogroms, les parents de Jodorowsky s'installent en effet à la fin des années 1920 dans cette sinistre petite ville portuaire, enclavée entre la montagne, les mines et la mer. Jaime, son père, est un homme en apparence complexe et impitoyable. Pétri de contradictions, cet ancien artiste de cirque, à la fois juif - mais profondément athée - et communiste, vouant un culte démesuré à Staline au point de se vêtir comme lui, tient à présent une boutique de lingerie avec sa femme : la "Casa Ukrania ». La mère du jeune Alejandro, Sara (Pamela Flores) est représentée comme une matrone à la poitrine opulente qui ne s'exprime qu'en chants lyriques. C'est au sein de ce couple que tout semble opposer, de cette famille à la fois déracinée et attachée à des valeurs indéfectibles, que Jodorowsky va recevoir une éducation stricte et parfois cruelle, tentant désespérément de se rendre digne de l'amour d'un père qui lui inflige les supplices les plus absurdes pour faire de lui un homme estimable. C'est l'exacte Tocopilla qu'il filme, avec tout ce qu'elle comporte et comportait d'êtres improbables comme la multitude de lépreux, les mineurs mutilés conduits à la décharge, ou encore le petit cireur de chaussures. Le réalisateur insiste en effet sur cette représentation de la réalité :

J'ai filmé Tocopilla telle qu'elle est aujourd'hui. En quatre-vingt années, elle n'a pas changé. La seule chose qui n'existait pas, c'était le magasin de mon père qui avait brulé. Alors je l'ai reconstruit exactement à l'identique. Et j'ai marché là où mes pieds d'enfants avaient marché. Mes pieds d'adulte ont marché dans les mêmes rues où je suis redescendu dans mon passé, vu par les yeux de l'enfant, plus surréaliste que ce qu'on appelle réalités ${ }^{31}$.

Dès lors, le cinéaste réalise par la fiction cinématographique les rêves respectifs de ses parents en faisant de sa mère une cantatrice lyrique et de son père un héros national. Plutôt que de dresser un simple décor, il s'agit surtout pour le cinéaste et les siens de réaliser une véritable "guérison familiale ${ }^{32}$ ». Devant ou derrière la caméra, c'est une grande partie de la "tribu» Jodorowsky qui s'implique. Brontis, qui avait fait ses premiers pas au cinéma à huit ans dans El Topo (1970) incarne de manière saisissante son grand-père, Jaime ; Alejandro endosse le rôle du narrateur, guidant son "Moienfant» puis son "Moi-jeune homme»; Axel (Cristobal) campe le personnage mystique de Théosophe, tandis qu'Adan se charge de composer la musique dans $L a$ Danza de la realidad avant d'incarner son père dans Poesía sin fin. Ainsi, l'œuvre de Jodorowsky, une «bombe atomique mentale ${ }^{33}$ " selon ses mots, lui permet d'entreprendre une forme de guérison familiale. Dans la droite lignée de la «psychomagie » dont il est le fondateur, thérapie consistant à guérir les problèmes de l'enfance, sa création cinématographique lui permet de retourner à la source de sa jeunesse, dans le lieu même où il a grandi, pour se réinventer. C'est une reconstruction qui part de la réalité mais permet de changer le passé.

20

Le filtre de l'imaginaire et de l'onirisme ne passe plus par le traitement technique de l'image, mais simplement par le contenu même du film. La Danza de la realidad offre la 
vision fantasmée d'un enfant qui vient sublimer une réalité pourtant bien présente, où seuls quelques effets spéciaux apparaissent comme autant de touches poétiques. À travers la représentation de cette réalité passée, Jodorowsky réalise les fantasmes familiaux par le biais d'une création artistique limitée en artifices. Par ailleurs, le réalisateur précise :

Le cinéma hypnotise mais doit montrer, comme Brecht l'a fait au théâtre, la distanciation. Il faut avoir en tête que l'on regarde un film, une œuvre d'art. Si les artifices sont visibles, ça n'a pas d'importance, c'est un film. En revanche, certains éléments doivent être vrais : la scène où l'homme urine, c'est réel ${ }^{34}$.

21 De même, Poesía sin fin présente des scènes à la tonalité surréaliste : la maison du jeune poète n'est meublée que d'une immense chaise disproportionnée qui n'est pas sans rappeler l'étonnant traitement de l'espace des surréalistes et notamment les récits poétiques de Boris Vian. Le personnage de Stella évoque de même un personnage fantasque et onirique; quant au Café Iris, qui mêle l'esthétique des tableaux de Magritte - avec les personnages austères en costume et à chapeau - aux décors des pièces beckettiennes - un espace épuré et froid, où les serveurs et les clients, outre leurs actions absurdes, semblent simplement attendre, ensemble, peut-être pour s'assurer de leur existence, que le temps passe -, il semble également être le produit d'une vision fantasmée du cinéaste. Ce n'est donc pas tant la technique que le filtre du souvenir, le plongeon dans le passé et la tentation de le modifier qui viennent déformer la réalité. Au cours de La Danza de la realidad se succèdent également des séquences réalistes voire crues et d'autres fantasmatiques et oniriques (lorsque la mer, blessée par une pierre, recrache des milliers de poissons), grotesques (avec le concours de chiens déguisés), ou qui tendent au cérémonial solennel et mystique (lorsque la mère guérit son époux de la peste ou qu'elle soigne avec ses urines).

Ainsi, nous retrouvons dans les créations cinématographiques de Jodorowsky l'esthétique surréaliste, tant par les images que par la visée, dans la mesure où son but est de nous libérer de nos carcans. D'une certaine manière, on retrouve entre l'œuvre du cinéaste et l'esthétique surréaliste cette même volonté de mener une révolte qui serait une exaltation de la liberté, une volonté d'affirmer, à travers la réalité, une autre vérité.

\section{Une quête du soi}

Les créations cinématographiques de Jodorowsky, visant à guérir son âme et à se réconcilier avec son père, apparaissent dès lors comme une quête: "Je pense que le pardon, ce n'est pas donner l'autre joue. Je pense plutôt qu'il faut comprendre pourquoi. C'est une fois que l'on a compris que l'on peut vivre. Le pardon, c'est la compréhension ${ }^{35}$. » Son père, Jodorowsky l'a souvent mis en scène de façon allégorique, notamment dans $\mathrm{El}$ Topo, et l'a longtemps considéré comme tyrannique. Mais dans $L a$ Danza de la realidad et Poesía sin fin, il puise dans l'enfance et l'onirisme la force d'affronter cette sombre vision, transformant la réalité à travers les yeux de son personnage enfant, devenu adulte. Dans les premières parties de ses derniers films se succèdent de pénibles moments de vie avec ce père sévère et intransigeant. La force de Jodorowsky est de reconsidérer tous ces événements à travers le prisme du fantasme, modulant la réalité pour plonger dans une aventure spirituelle. Par le biais de cette "danse » exaltante et déroutante, où réalité et imaginaire, tragique et légèreté, se mêlent, Jodorowsky emporte son spectateur dans une quête émouvante. La relation de 
ce fils avec un père dont il recherche désespérément l'amour devient réalité par le spectre du cinéma. La réconciliation avec le père, ou du moins son souvenir, permet alors à l'enfant devenu homme de se reconstruire. Jodorowsky adulte guide d'ailleurs le jeune Alejandro tout au long du film, observant et conseillant l'enfant qu'il était pour le mener sur la voie d'une élévation spirituelle. Dans Poesía sin fin, cette quête spirituelle et de réconciliation est dépassée par la quête de soi. Ses premiers vers, adressés à son cousin Ricardo, expriment bien cette volonté de «Revenir à ce qu'[il a] toujours été ». Plus tard, il exprimera également cette nécessité : «Je suis bien plus mes traces que mes pas ». Et la fin du film propose une réflexion sur la finalité de son être, et la crainte de l'oubli après la mort.

Pour Jodorowsky, «l'art doit être plus que l'art, il faut créer autre chose qu'un spectacle capable d'amuser ou de susciter l'admiration ${ }^{36}$.» Solennellement, la pénétrante voix du réalisateur résonne aux oreilles de son "Moi-passé » :

Toi et moi n'aurons été que des souvenirs, jamais une réalité. Quelque chose est en train de nous rêver. Donne-toi à l'illusion. Vis ! [...] Tout ce que tu seras, tu l'es déjà. Ce que tu cherches est déjà en toi. Réjouis-toi de tes chagrins. Grâce à eux, tu arriveras à moi.... ${ }^{37}$

Mon père, ne me donnant rien, tu m'as tout donné. Tu m'as donné la force de supporter le monde où la poésie n'existe plus. J'ai appris à être, j'ai appris à aimer, j'ai appris à créer, j'ai appris à vivre ${ }^{38}$.

C'est un Jodorowsky plus apaisé, à l'image d'un vieux sage, et plus optimiste peut-être, qui apparaît. Il n'est plus question, comme dans El Topo, d'affirmer la nécessité d'agir violemment sur le spectateur pour lui faire prendre conscience de la léthargie intellectuelle et spirituelle dans laquelle il se conforte. À l'époque, le héros se trouve dans l'acceptation permanente de la souffrance et échoue dans sa quête initiatique et sa recherche de vérité, refusant un monde corrompu. Aujourd'hui, l'idée d'une société vouant à l'échec l'émancipation de l'homme en l'enfermant dans le misérabilisme de sa condition - thème déjà développé dans La Montagne sacrée (La Montaña sagrada, 1973) laisse place à l'exaltation des potentialités de l'être, à la réconciliation d'un homme avec son enfance. Ainsi, La Danza de la realidad et Poesía sin fin dressent le tableau d'une existence à la fois invraisemblable et incroyablement authentique, d'où se dégage un insatiable désir de vivre: «La vieillesse n'est pas une humiliation, elle permet de se détacher de tout, [...] de devenir un papillon resplendissant de lumière ${ }^{39}$. " Jodorowsky emporte alors son spectateur dans un hymne à l'enfance, à la création, et une quête émouvante, celle de soi, et de la connaissance, dans la lignée des créations paniques.

\section{NOTES}

1. Ce texte est un prolongement d'une réflexion menée dans un précédent article : «Chemins de traverse ", L'Intermède [en ligne], 8 septembre 2013, http://www.lintermede.com/cinema-ladanza-de-la-realidad-jodorowsky-autobiographie.php. 
2. Alejandro Jodorowsky, "Conférence donnée lors de la projection de La Danza de la realidad (Forum des Halles, “La Quinzaine des réalisateurs", juin 2013 », dans La Danza de la realidad [DVD], Pathé, 2015.

3. Alejandro Jodorowsky "Vers l'éphémère panique...", dans Fernando Arrabal, Panique. Manifeste pour le troisième millénaire, Paris, Éditions Punctum, 2006, p. 92.

4. Ibid., p. 86.

5. Si Jodorowsky a découvert les richesses des éphémères très tôt, dès 1948 , considérant la création de fêtes comme la finalité de l'expression artistique, c'est le 24 mai 1965 qu'il met en application ses théories avec Arrabal, et monte le plus important spectacle panique sous le titre «Le groupe panique international présente sa troupe d'éléphants » lors du deuxième festival de la Libre Expression fondé par Jean-Jacques Lebel. Cette représentation regroupe trois éphémères: La Femme nouvelle de Topor, Les Amours impossibles d'Arrabal et Auto sacramental de Jodorowsky. Elle dure près de six heures, met en scène une soixantaine d'acteurs et réunit une quantité colossale d'objets organiques - des têtes d'animaux morts, cinq cent kilos de viande animale, des centaines de litres de sang chaud et de liquides divers, trois cent tortues -, ainsi que de nombreux costumes et accessoires monstrueux et fantastiques. Si une partie de la critique reste caustique à l'égard de ces performances, d'autres en revanche, comme le rapporte Arrabal, semblent avoir vécu lors de cette cérémonie une expérience déroutante, entre répugnance et fascination : "C'est au milieu de la richesse la plus surprenante, dans un théâtre de "choc", qu'on célébra la cérémonie de l'éphémère panique. Pendant près de cinq heures le public assista, au milieu d'une tension quasi hystérique, au développement d'une représentation qui a par la suite été qualifiée de "spectacle le plus transcendant du siècle". Sur scène, on baignait un "couple amoureux" dans des litres et des litres d'huile et de vinaigre, on extirpait du "père" une à une, toutes ses viscères - sexe et testicules inclus -, que quelqu'un faisait aussitôt frire dans une poêle et les offrait au public comme des steaks; la sadique fouetta réellement le masochiste jusqu'à transformer son dos en plaie béante; la scène et le théâtre furent remplis de poussins nouveaunés, de couleuvres, de tortues, de petits oiseaux, de pigeons, de phoques, de viande de bœuf... Le taureau séduisit la princesse ; pendant qu'il la séduisait il l'aspergeait de mousse, qui jaillissait "spermatiquement" d'une de ses cornes, préalablement frottée et tripotée par la main de cette demoiselle. Un groupe de rock - celui de Vince Taylor - jouait très fort et, par moments, il s'arrêtait pour jouer la plus mélancolique des mélodies : les chants d'un mariage juif. Un chien se suicida sur scène ; six danseuses se baignèrent toutes nues sur scène; le rival d'un authentique poète rasa les poils pubiens de la femme de ce dernier. Tout cela avait un argument qui servait de base, comme dans les partitions de jazz. Tout cela avait été très répété, mais on donna à tout le monde la liberté de créer. Jamais on n'entendit à Paris une ovation aussi formidable que celle de ce soir-là », nous traduisons, Fernando Arrabal, Teatro Panico, Madrid, Catédra, 1986, p. 27.

6. Ibid., p. 91.

7. Alejandro Jodorowsky "Vers l'éphémère panique...», dans Fernando Arrabal, Panique. Manifeste pour le troisième millénaire, op. cit., p. 83.

8. Si l'on peut trouver des similitudes entre l'attitude des artistes italiens de l'Arte povera - l'Art pauvre, expression utilisée par Germano Celant en septembre 1967 pour intituler une exposition présentée à Gênes -, dont les expériences se multiplient particulièrement entre 1966 et 1969, et l'esthétique panique, les artistes du groupe ne vont pas aussi loin dans le positionnement politique de cette démarche artistique qui consistait à défier l'industrie culturelle ainsi que la société de consommation. Nous retrouvons cependant chez Jodorowsky ou même Arrabal une même importance accordée au processus artistique et au dérisoire, dans leur conception de la mise en scène.

9. Alejandro Jodorowsky "Vers l'éphémère panique...», dans Fernando Arrabal, Panique. Manifeste pour le troisième millénaire, op. cit., p. 93.

10. Ibid. 
11. Ibid., p. 94.

12. Jean-Paul Coillard, De la cage au grand écran, entretien avec Alejandro Jodorowsky, Paris, Editeur K-ïnite, 2009, p. 113.

13. Ibid.

14. Alejandro Jodorowsky, dans Michel Larouche, Alejandro Jodorowsky, Cinéaste panique, Montréal, Les Presses de l'Université de Montréal, 1985, p. 172.

15. Alejandro Jodorowsky, « Panique et poulet rôti... », Panique, Manifeste, op. cit., p. 65.

16. Les fêtes organisées dans la maison des artistes où chez le jeune Alejandro mettent également en scène des personnages hauts en couleur, dont l'identité artistique se manifeste par les vêtements, l'apparence ou la gestuelle, contrairement aux habitants, souvent affublés de masques blancs inexpressifs.

17. Titre d'un tableau de Goya et d'un roman d'Arrabal.

18. Alejandro Jodorowsky, "Conférence donnée lors de la projection de La Danza de la realidad (Forum des Halles, "La Quinzaine des réalisateurs”, juin 2013 », dans La Danza de la realidad [DVD], op. cit.

19. Ibid.

20. Peter Brook, Le théâtre 1968-1, Cahiers dirigés par Arrabal, Paris, Christian Bourgois, 1968, p. 14-15.

21. La pratique de leur art, le piano, la peinture ou la danse, se fait pour ces artistes tout en démesure. Exaltés, ils semblent tomber dans un état de transe créatrice qui fait de l'œuvre musicale, plastique ou chorégraphique, une véritable performance physique qui engage visuellement le corps.

22. Conception développée par Antonin Artaud dans "Le théâtre et la peste ", Le Théâtre et son double, Paris, Gallimard, 1964.

23. Alejandro Jodorowsky, dans Jean-Paul Coillard, De la cage au grand écran, entretien avec Alejandro Jodorowsky, op. cit., p. 138.

24. Alejandro Jodorowsky, «Conférence donnée lors de la projection de La Danza de la realidad (Forum des Halles, “La Quinzaine des réalisateurs", juin 2013 », dans La Danza de la realidad [DVD], op. cit.

25. Ibid.

26. René Girard, La Violence et le sacré, Paris, Bernard Grasset, 1993.

27. Michel Viegnes, "Les monstres : une espèce pas du tout menacée », dans Anne-Laure Milcent, L'Inquiétante Étrangeté des monstres, monstruosité, altérité et identité dans la littérature française, XIX ${ }^{\text {- }}$ $\mathrm{x} \mathrm{X}^{e}$ siècle, Dijon, Editions Universitaires de Dijon, 2013, p. 24.

28. Alejandro Jodorowsky, L'Arbre du dieu pendu, trad. fr. Mara Hernandez et René Solis, Paris, Métailié, 1996, p. 10.

29. Michel Larouche, Alejandro Jodorowsky, Cinéaste panique, op. cit., p. 19-20.

30. Alejandro Jodorowsky, "Conférence donnée lors de la projection de La Danza de la realidad (Forum des Halles, "La Quinzaine des réalisateurs", juin 2013 », dans La Danza de la realidad [DVD], op. cit.

31. Ibid.

32. Alejandro Jodorowsky, entretien avec Olivier Père [en ligne], 18 mai 2013, URL : https:// www.arte.tv/sites/olivierpere/2013/05/18/cannes-2013-jour-4-la-danse-de-la-realitedalejandro-jodorowsky-quinzaine-des-realisateurs/ (dernière consultation le 09/09/2019).

33. Ibid.

34. Alejandro Jodorowsky, "Conférence donnée lors de la projection de La Danza de la realidad (Forum des Halles, “La Quinzaine des réalisateurs", juin 2013 », dans La Danza de la realidad [DVD], op. cit.

35. Ibid. 
36. Alejandro Jodorowsky, entretien avec Olivier Père [en ligne], 18 mai 2013, URL : https:// www.arte.tv/sites/olivierpere/2013/05/18/cannes-2013-jour-4-la-danse-de-la-realitedalejandro-jodorowsky-quinzaine-des-realisateurs/ (dernière consultation le 09/09/2019).

37. Alejandro Jodorowsky, La Danza de la realidad [DVD], op. cit.

38. Alejandro Jodorowsky, Poesía sans fin [DVD], Blaq Out, 2017.

39. Ibid.

\section{RÉSUMÉS}

Cinéaste, mime, scénariste de bandes dessinées, romancier, poète, co-fondateur du groupe Panique ou encore théoricien de tarot divinatoire, Alejandro Jodorowsky est et se dit " multiple». Cette esthétique du multiple et du rêve se retrouve dans ses deux derniers films La Danza de la realidad et Poesía sin fin. Notre étude se propose d'identifier les points de convergence entre ces deux œuvres et l'esthétique panique. Afin d'étudier les dialogues qui se tissent au cœur de ces films entre diverses formes artistiques et influences culturelles ou biographiques, il s'agira de voir que le cinéma est devenu pour Jodorowsky un lieu privilégié des manifestations paniques, qui, tendant à la "fête-spectacle", englobent toutes les formes d'art et laissent place à la pluralité. Puis nous tâcherons de voir en quoi Jodorowsky livre une vision onirique d'un passé où s'entremêlent réalité et fantasmes, dans la lignée de l'esthétique surréaliste, par le biais d'une création artistique épurée.

Filmmaker, mime artist, comic book scriptwriter, novelist, poet, co-founder of the Panique group or divinatory tarot theorician, Alejandro Jodorowsky is and also self-defined as "a pluralist". This aesthetics of plurality and dream is found within his two last movies La Danza de la realidad and Poesía sin fin. Our study is focused on highlighting the convergences between these two films and the panic aesthetic. In order to study the relations developed throughout the films between many artistic forms and cultural or biographical influences, we will first intend to show that cinema has become for Jodorowsky a very special space for panic shows which, tending to "Party-Performance", include every forms of art and gives expression to plurality. Then, this work will show how Jodorowsky brings an oneiric sight of his own past while mixing up reality and fantasy, in line with the surrealist aesthetic, by offering a purified artistic creation.

\section{AUTEUR}

\section{ÉMILIE COMBES}

Enseignante en Lettres Modernes/Théâtre (dans le secondaire et chargée de cours à l'Université Paris III-Sorbonne Nouvelle), docteure en Littératures Françaises et directrice de la rédaction du site d'actualité culturelle L'Intermède.com.Ses recherches portent essentiellement sur l'œuvre de Fernando Arrabal mais s'étendent aussi à la poétique théâtrale et à la création scénique contemporaine. Depuis 2012, elle publie des articles critiques sur les créations théâtrales contemporaines pour L'Intermède. Dans le cadre de ses recherches, elle a participé à plusieurs publications et communications : lors d'un Festival d'Art contemporain, elle a donné une conférence en décembre 2014 intitulée «Arrabal et le Panique » et a publié des articles sur le 
sujet : «L'exaltante expérience du Panique : de la terreur à la fascination », pour L'Atelier du roman, Flammarion $n^{\circ} 74$, en juin 2013 ; « De l'anarchisme à Panique : Arrabal, libertaire mystique », chez Asian Culture Publishing, en avril 2013, dans le cadre de la traduction des Lettres à Franco, Staline et Castro, de Fernando Arrabal ; « Le dépassement de soi par la transgression des interdits chez Arrabal : quand l'érotisme brutal devient vecteur de communion avec le sacré », paru dans la revue Aletria, Revista de Estudos de Literatura, en 2017. Ses travaux de thèse : « Le théâtre Panique de Fernando Arrabal, science de l'essence de la confusion », sont à paraître chez Classique Garnier. 